\title{
Discovery of Low Mass Objects in Taurus
}

\author{
W. J. Forrest, Z. Ninkov, J. D. Garnett \\ University of Rochester \\ M. F. Skrutskie \\ University of Massachusetts \\ M. Shure \\ University of Hawaii
}

\begin{abstract}
An infrared (2.2 $\mu \mathrm{m}, K$-band) search of small regions (25" square) near 26 members of the Taurus star-forming association has revealed $20 \operatorname{dim}(K=13-16 \mathrm{mag})$ stellar objects near 13 of them. Of these 20 objects, 9 are exceptionally red. It is argued that these 9 are probably also Taurus members. From the luminosities $\left(0.8-4 \times 10^{-3} L_{\odot}\right)$ and ages (estimated at $10^{6}$ years), masses can be determined by reference to theoretical low-mass cooling curves. The masses are in the range 0.005-0.015 $M_{\odot}$, i.e. low-mass brown dwarfs. Proper motion studies of 7 of the objects visible on the POSS plates conducted by Burton Jones establish that 4 are highly probable Taurus members while 1 is a possible member.
\end{abstract}

\section{Introduction}

The theory of self-gravitating gaseous bodies predicts that below a mass of about 0.08 $M_{\odot}$, the central temperatures will never be high enough to initiate stable nuclear burning. These sub-stellar objects, which subsist on gravitational energy and cool eternally, have been dubbed brown dwarfs (Tarter, 1986). To date, there are no unambiguous indentifications of a bona-fide brown dwarf, though there are several candidates which are either low mass stars or high mass brown dwarfs, depending on their precise ages. It is of great interest to know if brown dwarfs do exist in the galaxy and, if so, how many there are and what are their masses. A sufficient population of brown dwarfs could represent a large mass which is dim visually. This would have enormous implications for the "missing mass" problem in our galaxy and perhaps others.

Previous searches for brown dwarfs have concentrated on objects ranging in age from $\sim 10^{8}$ to $10^{10}$ years. At these advanced ages, brown dwarfs will be cool and dim, especially low mass brown dwarfs. Therefore such searches require high sensitivity to cool objects and will be most sensitive to higher mass brown dwarfs. At very young ages, however, even relatively low mass brown dwarfs of $0.01 M_{\odot}$, will be relatively luminous. Furthermore, because of the nature of the Hayashi evolutionary tracks, dominated by convection internally and $\mathrm{H}^{-}$radiative opacity at the surface, the effective temperatures will be relatively high, around $2500-3500 \mathrm{~K}$ in the early stages of evolution. For instance, a $0.01 M_{\odot}$ brown dwarf $10^{6}$ years old will have a luminosity of $\sim 1.6-1.8 \times 10^{-3} L_{\odot}$ and an effective 
temperature $\sim 2300-2600 \mathrm{~K}$ (Nelson et al. 1986, Lunine et al. 1989).

Based on these ideas, we searched in the vicinities of $26 \mathrm{~T}$-Tauri stars in the Taurus star-forming association. A total of $20 \mathrm{dim}$ (apparent $K$ magnitude 13-16) objects were discovered near 13 of the $T$-Tauri stars. Of these 20 , at least 9 were exceptionally red. We argue that it is extremely unlikely that the red objects could be foreground or background stars and are therefore probably also members of Taurus. Assuming ages equal to the average age of Taurus members $\left(10^{6}\right.$ years $)$ and using the above theoretical evolutionary curves, we estimate masses of $0.005-0.015 M_{\odot}$ based on the observed luminosities.

The key question of true Taurus membership has received strong support from the proper motion studies of Burton Jones (1989, private communication). Of the seven objects visible on the mid-1950's POSS red plates, he has found 4 to have proper motions which indicate Taurus membership and rule out a background star. We conclude that the bulk of the red stars really are Taurus members and therefore low mass brown dwarfs as calculated above.

\section{Observations}

Infrared observations of 26 T-Tauri stars in Taurus were made at the NASA $3.0 \mathrm{~m}$ Infrared Telescope Facility on Mauna Kea on 6 nights in September, 1988 using the Rochester $58 \times 62 \mathrm{InSb}$ infrared camera. (This project had not been granted offcial observing time, so a small amount of time, amounting to one night total, was borrowed from other approved projects for this run.) Our procedure was to place the T-Tauri star in the center of the $25^{\prime \prime}$ square field-of-view of the camera. Then the telescope was nodded to presumed blank sky, usually 100-200" S, to acquire a background frame. The telescope was then nodded back to the T-Tauri star and 2 exposures of 33 seconds each in the photometric $K$-band $\left(\lambda_{o}=2.23 \mu \mathrm{m}, \Delta \lambda=0.41 \mu \mathrm{m}\right)$ were acquired. The images were inspected by subtracting the background frame and displaying them on a video monitor with display levels set to reveal the dimmest possible object. A stellar object near the T-Tauri star was seen as a bright (positive) image, while a stellar object in the sky frame was seen as a dark (negative) image. We estimate a limiting magnitude of circa 16 for definite detection of positive stars and somewhat brighter for negative stars.

If a positive star was seen, measurements in the $H\left(\lambda_{0}=1.65 \mu \mathrm{m}, \Delta \lambda=0.32 \mu \mathrm{m}\right)$ and $J\left(\lambda_{o} \cong 1.25 \mu \mathrm{m}, \Delta \lambda \cong 0.21 \mu \mathrm{m}\right)$ bands were also acquired. In addition, if the original telescope position placed the newly discovered object in a bad part of the array, either near the edge or near the crack in the southeast corner, the telescope was moved to re-position the source more optimally. In most cases, a negative star, i.e. one present in the distant sky beam, was not followed up with $J$ and $H$ photometry unless a positive star was also present. On some occasions, our sky beams were positioned only $24^{\prime \prime}$ from the main beam. In these cases, negative stars were measured in all bands.

A typical $2.23 \mu \mathrm{m}$ image is shown in figure 1 . The $K \sim 13 \mathrm{mag}$ stellar object $8^{\prime \prime} S$ of $\mathrm{LkCa} 4$ was first seen. Moving the telescope for better viewing revealed the second $K \sim 14$ mag stellar object $13^{\prime \prime} W$ of $\mathrm{LkCa} 4$. In this case, a $75^{\prime \prime}$ square region surrounding $\mathrm{LkCa}$ 4 was also surveyed, to a limit of $K=15-16 \mathrm{mag}$. No further stellar objects were found. 
The images were processed as follows to derive photometric magnitudes. First, as discussed by Forrest et al. (1989, this conference), the output of the array is inherently non-linear. Data, both source and background images, were therefore first linearized as described in Forrest et al. (1989). After background subtraction, the pixel-to-pixel nonuniformities were eliminated through division by a flat field. We considered candidate flat fields (linearized) from the blue morning or evening sky and the inside of the dome illuminated by five $1000 \mathrm{~W}$ incandescent light bulbs. There was very little difference between any of the flat fields taken throughout the run. We tested the photometric performance of the flat fields by imaging standard stars at 25 grid positions on the array. Using a combination of dome and blue sky flat fields, the best photometric performance was a standard deviation of $4.5 \%$ of the average. As discussed in Forrest et al. (1989), we attribute a large component of the variation to pincushion distortion in our reimaging optics. After correcting the flat field for this effect, the variation improved to $2.6 \%$ at $K$. The uncertainty due to flat fielding will be somewhat higher at $J$ and $H$.

Standard stars were observed throughout each night for calibration purposes. Measurements in a synthetic octagonal aperture $4.6^{\prime \prime}$ in diameter indicated quite stable values in the $J, H$, and $K$ bands. The companions were often very faint, ranging down to a $J$ magnitude of 17. For accurate photometry, a method of estimating the total signal from the star without contribution from nearby sky signal was developed. An octagonal aperture $3.8^{\prime \prime}$ in diameter was centered on the companion. The outer annulus $0.42^{\prime \prime}$ (one pixel) wide was used to estimate the sky level. This surface brightness level (counts per pixel) was subtracted from the inner octagon, $2.9^{\prime \prime}$ in diameter, to give the total star signal. This method of differential photometry is dependent on the seeing, which will smear true star signal into the sky annulus. Measurements on the standard stars indicated a $\pm 2 \%$ uncertainty at $H$ and $K$ and $\pm 5 \%$ uncertainty at $J$ due to this effect.

We estimate a net uncertainty $\pm 10 \%$ in the $K, H, J$ fluxes and colors. To this must be added uncertainty due to detector noise for the fainter sources. Tests of the accuracy of the photometry and colors were generally favorable. For a red standard, we used Giclas 77-31 (Elias et al. 1982) with $K=7.84, J-H=0.58$ and $H-K=0.32$. Calibrating this in the same manner as the companion stars gave $K=7.90, H-K=0.26$ and $J-H=0.56$, well within our quoted $10 \%$ uncertainties. For dim standards we observed the white dwarfs H 27 and VR 16 from Zuckerman and Becklin (1987,ZB). For H 27 we got $J=14.68 \pm 0.15, H=14.71 \pm 0.1, K=14.72 \pm .14$ while $\mathrm{ZB}$ quote $J=14.70, K=14.90$. The $J$ magnitude is satisfactory but the $K$ magnitudes differ by 0.18 , which is somewhat larger than our estimated uncertainty of $0.14 \mathrm{mag}$. This may indicate a slight problem in our photometry, or it could indicate that $\mathrm{H} 27$ has an infrared excess similar to the white dwarf LB 1497 studied by ZB. For VR 16, we found the white dwarf had a dim companion $4.4^{\prime \prime}$ to the $W$ (as well as a star comparable in brightness to the white dwarf $6^{\prime \prime} \mathrm{W}$ in our $100^{\prime \prime} \mathrm{S}$ sky beam). For the white dwarf we derive $J=14.58, H=14.61$ and $K=14.74$, somewhat brighter than ZB's $J=14.64, K=14.85$. The $4.4^{\prime \prime} \mathrm{W}$ companion was $J=16.14, H=15.97, K=15.86$. ZB used $12^{\prime \prime}$ diameter aperature, which should have included the $4^{\prime \prime} W$ companion. However, the sum of the white dwarf and the $4^{\prime \prime} W$ companion is brighter than ZB's measurements by $0.3-0.4$ magnitudes, which is well outside the uncertainties. (We conclude that $\mathrm{ZB}$ probably missed this companion.) If the 
$4^{\prime \prime} \mathrm{W}$ companion is also in the Hyades, its absolute $K$ magnitude would be $M_{K}=12.6$, which would be the dimmest known main sequence star. Since it is not outstandingly red, we suggest it may be a cool white dwarf. A typical background star would be a $\mathrm{K} 1$ main sequence star reddened by $A_{K}=0.22$. This appears incompatible with the observed colors of the $4^{\prime \prime} W$ companion.

We conclude there is no systematic inaccuracies in our $J, H, K$ photometry of $\operatorname{dim}$, red objects exceeding 0.1 in the magnitudes or colors. The photometric results for our objects are given in Table 1. In Table 2 we list the T-Tauri stars without any nearby objects.

The plate scale, orientation, and optics distortion of our camera were determined by measuring the star pairs $\gamma$ Ari and $\gamma$ Del and the stars $\ominus^{1} A, B, C$, and $D$ in the Trapezium. The average plate scale at $K$ was $0.42^{\prime \prime}$ /pixel and the columns of the array were tilted $0.3^{\circ}$ from north. By modeling the pincushion distortion, an accuracy of $0.5 \%$ in plate scale and rotation was achieved over the whole array. The position of the companions relative to the T-Tauri stars were measured by calculating the centroid in the same aperature used for photometry. The resulting offsets, appropriate for the 1988.8 epoch are given in Table 1.

\section{Discussion}

We don't expect that all of the 20 objects discovered here are members of the Taurus star-forming association. To predict the number of background stars expected, a star count model based on the method of Elias (1978 a \& b) was developed. The star densities given by Elias (1987a) were used. These were supplemented for main sequence spectral types later than K3 using the $V$ luminosity function of D'Antona and Mazzitelli (1986). This was transformed to a $K$ luminosity function using the relationship $M_{K}=0.521 M_{V}+0.80$, which was found to fit the range $M_{V}=4.4$ to 17 . This relationship is derived from Liebert and Probst's (1987) fig. 2 for low mass stars and Allen (1973) for higher mass stars. A further extension of the Elias model was the inclusion of a screen of extinction $A_{K}=0.2$ mag. at $140 \mathrm{pc}$ to account for the average extinction through Taurus of $A_{V} \simeq 2$. The model was normalized to the $K$ star count data of Elias $(1978 \mathrm{~b})$ in Taurus by variation of the radial scale length $\alpha$ (Elias $1978 \mathrm{a}$ ). The resulting value of $\alpha=3 \mathrm{kpc}$ gave a predicted value of 10.7 stars per square degree for $K<7.5$, in adequate agreement with the actual counts of 11 . The cumulative number of stars brighter than $K=15.5$ from this model is 3033 stars/square degree. For the observed counts, we have taken only the companions seen at the initial telescope setting, with the T-Tauri star centered on the array. (We do not include additional stars revealed when the telescope was moved or stars appearing in our sky beams). We found 9 such objects brighter than $K=15.5$ in our search of 26 , $\left(25^{\prime \prime}\right)^{2}$ fields. The model predicts 3.7 , a factor of 2.4 lower. Therefore, we anticipate that somewhat less than half of our objects may be field stars, while the rest are likely Taurus members, based on the star counts alone.

To select the likely Taurus members, we consider the infrared colors $J-H$ and $H-K$. The colors from table 1 are plotted in figure 2. Also shown are the colors of main sequence stars, the colors generated by interstellar extinction of $A_{V}=2 \mathrm{mag}$, and the colors of 
2000-3500 $K$ black bodies. The star count model predicts that the average field star will be a $\mathrm{K} 1$ main sequence star $1.3 \mathrm{kpc}$ distant with $A_{K}=0.32$ (corresponding to $A_{V}=3.2$ mag.). Such a star would have colors $J-H=0.75$ and $H-K=0.33$. Nine of our objects have red colors, which differ significantly from these predicted colors, suggestive of low mass objects in Taurus. We suggest these objects are likely Taurus members, based on their colors and the large number found.

Red giant stars have colors similar to our objects, but a red giant would have to be $\sim 200 \mathrm{kpc}$ away to appear at $K=15$ magnitude. Reddened late $\mathrm{M}$ dwarfs would have colors similar to our companions. However, from the star count model described above we expect only $1 \mathrm{M}$-dwarf in our survey. Reddening in Taurus which exceeded the 0.2 mag. $A_{K}$ (2 mag. $\left.A_{V}\right)$, which we have already included, could cause a $\mathrm{G}$ to $\mathrm{K}$ background star to appear as red as our objects. However detailed star counts in Taurus by Cernicharo and Bachiller (1984) show a maximum of $A_{V}=4$ at the $\mathrm{LkCa} 5$ position and considerably less for the other objects. Therefore, this is not plausible either, since a value of $A_{V}>4$ in Taurus would be necessary to account for the observed colors.

A very strong test of Taurus membership is proper motion. Members of the Taurus star forming region have typical proper motions of $2.3^{\prime \prime} /$ century in P.A. $164^{\circ}$ east of north. (Jones and Herbig 1979). A typical background star $1.3 \mathrm{kpc}$ distant will have very small proper motion, $\sim .26^{\prime \prime}$ /century. Burton Jones (1989) has inspected the POSS plates from the mid 1950's and finds about half of our objects can be seen on the red plate. The others are either not visible or too close to the primary to be seen. For 7 of the objects with proper motion data, 4 definitely have a proper motion characteristic of Taurus members and inconsistent with background stars. These are almost certainly Taurus members. Of the others, one is indeterminate, one has high proper motion $\left(\sim 20^{\prime \prime} /\right.$ century $)$ and one has a low proper motion consistent with a background star (table 1). We conclude that a large fraction of our 11 most likely candidates actually are members of the Taurus star forming association.

In order to estimate the masses of the dim, red Taurus members, we compare the observed luminosities to those predicted by the theoretical evolutionary models of Nelson et al. (1986) and Lunine et al. (1989). Using the distance to Taurus of $140 \mathrm{pc}$, the luminosities are $0.8-4 \times 10^{-3} L_{\odot}$. For an age of $10^{6}$ years, which is the average age of the previously known Taurus members, the inferred masses are $0.005-0.015 M_{\odot}$, i.e. low mass brown dwarfs. The ages would have to exceed $10^{8}$ years for these objects to be stars $\left(M>0.075 M_{\odot}\right)$. This is counter to experience in Taurus, where the average age is only $10^{6}$ years and star formation apparently began in earnest only $10^{7}$ years ago (Jones and Herbig, 1979, Hartmann et al, 1987). Furthermore, the lifetime of star-forming molecular clouds is believed to be only $10^{7}-10^{8}$ years.

\section{Conclusions}

We conclude that Taurus harbors a considerable number of low-mass objects, typically $0.01 M_{\odot}$ or $10 M_{\text {Jupiter }}$, which could be described as low mass brown dwarfs or high mass planets. Detailed studies of the low mass objects identified here are necessary to better 
understand this new type of object.

Spectroscopic studies can lead to an understanding of the atmospheric structure. This will allow determination of the effective temperatures which are needed to place them on the Hertzsprung-Russell diagram and to compare them with theoretical evolutionary models of such objects. It may also be possible to determine the surface gravities, which will give a direct estimate of the masses. For the objects which can't be seen on the POSS plates for proper motion studies, the spectra will be needed to definitely establish Taurus membership. $K$-band spectra will reveal the $\mathrm{CO}$ absorption $(>2.3 \mu \mathrm{m})$ and $\mathrm{H}_{2} \mathrm{O}$ absorption $(1.9 \mu \mathrm{m}$ and $2.7 \mu \mathrm{m})$ if the object is actually a $2000-3000 \mathrm{~K}$ substellar object on its Hayashi convective track, rather than a reddened G-K background star.

Further survey observations are necessary to establish how widespread this phenomenon is. If such objects are found throughout the extensive star-forming regions in Taurus, they could represent a heretofore unknown but dynamically important mass component of our galaxy.

\section{References}

Allen, C. W. 1073 "Astrophysical Quantities" (The Athlone Press, London).

Cernicharo, J. and Bachiller, R. 1984 A \& A Supp, 58, 327

D'Antona, F. and Mazzitelli, I. 1986 Astron. Ap., 162, 80.

Elias, J. H. 1978a Ap J, 223, 859.

Elias, J. H. 1978b Ap J, 224, 857.

Elias, J. H., Frogel, J. A., Matthews, K. and Neugebauer, G. 1982 AJ, 87, 1029.

Forrest, W. J., Pipher, J. L., Ninkov, Z. and Garnett, J. D. 1989 (This conference prodeeding).

Hartmann, L. W., Soderblom, D. R., and Stauffer, J. R. 1987 AJ, 93, 907.

Herbig, G. H. and Bell, K. R., 1988 "Third Catalog of Emission-Line Stars of the Orion Population", Lick Obs. Bull. No. 1111 (U. Calif.)

Jones, B. F. 1989 private communication.

Jones, B. F. and Herbig, G. H. 1979, AJ, 84, 1872.

Liebert, J. and Probst, R. G. 1987 Am. Rev. Astron. Ap, 25, 473.

Lunine, J. I., Hubbard, W. B., Burrows, A. S., Wang, Y.-P., and Garlow, K. 1989 ApJ, 338, 314.

Nelson, L. A., Rappaport, S. A. and Joss, P. C. 1986 ApJ, 311, 226.

Tarter, J. C. 1986 in "Astrophysics of Brown Dwarfs" ed. Kafatos, Harrington and Maran (Cambridge U. Press, Cambridge) p. 121.

Zuckerman, B. and Becklin, EE 1987 ApJ (Letters), 319, L 99 (ZB). 

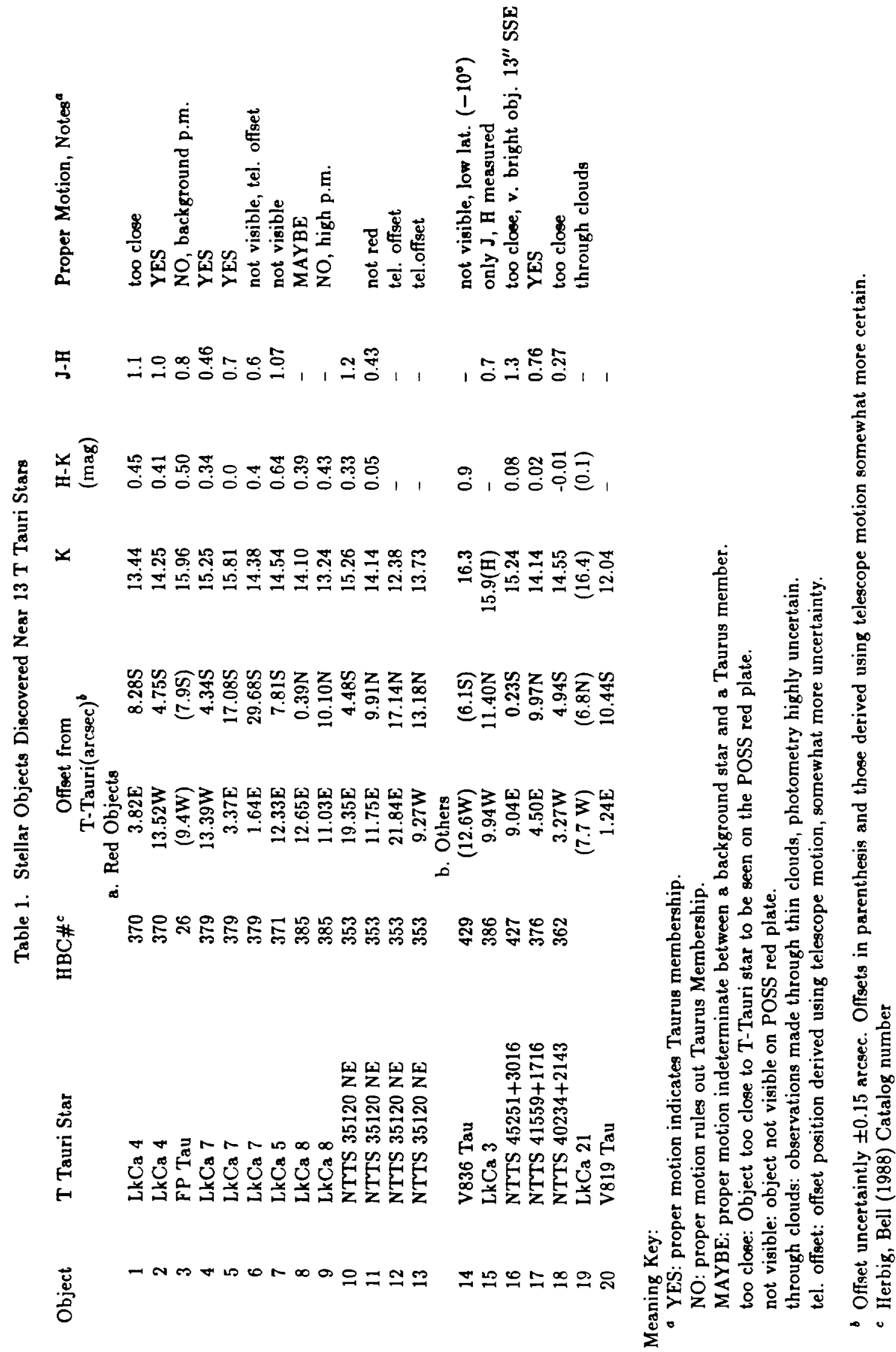


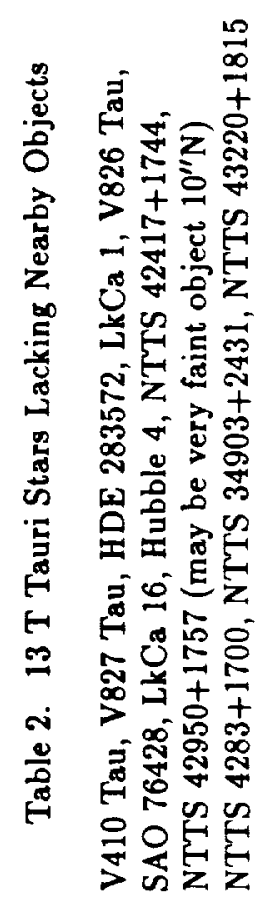




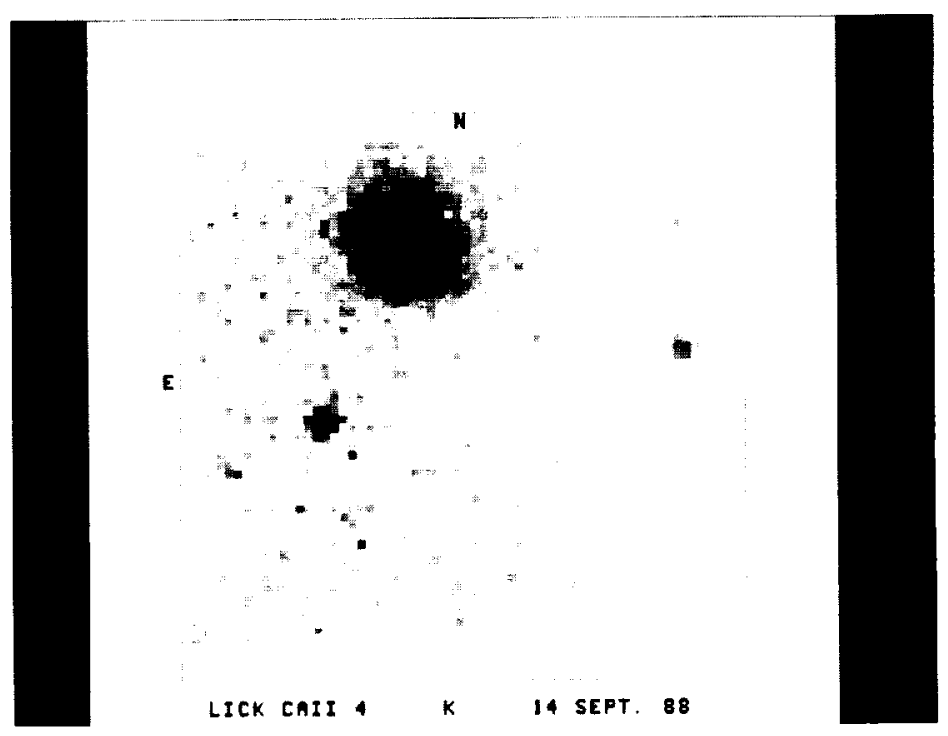

Figure $12.2 \mu \mathrm{m}(\mathrm{K})$ image of a $24^{\prime \prime} \times 26^{\prime \prime}$ region near the T Tauri star LkCa 4 made on 14 September 1988. North is up and East is to the left. The T Tauri star is the bright object near the top. The dim object to the right (west) can also be seen on the POSS red plate no. 1454 from 23 October 1955 . Its proper motion $\left(2.7^{\prime \prime} /\right.$ century E and $2.2^{\prime \prime}$ / century S (Jones, 1989) is consistent with that of LkCa 4 and other Taurus members but inconsistent with a distant background star. It is therefore a highly probable Taurus member. Its luminosity derived from the measured infrared fluxes is $2 \times 10^{-3} L_{\odot}$. Its mass is inferred to be $\simeq 0.01 M_{\odot}$ based on an estimated age of $10^{6}$ years. The brighter object to the left and below $\mathrm{LkCa} 4$ is too close to be seen on the POSS plates and, therefore, its proper motion is unavailable at this time. Its red colors indicate probable Taurus membership. If this is confirmed spectroscopically, its luminosity would be $4 \times 10^{-3} L_{\odot}$ and its inferred mass about $0.015 M_{\odot}$. 


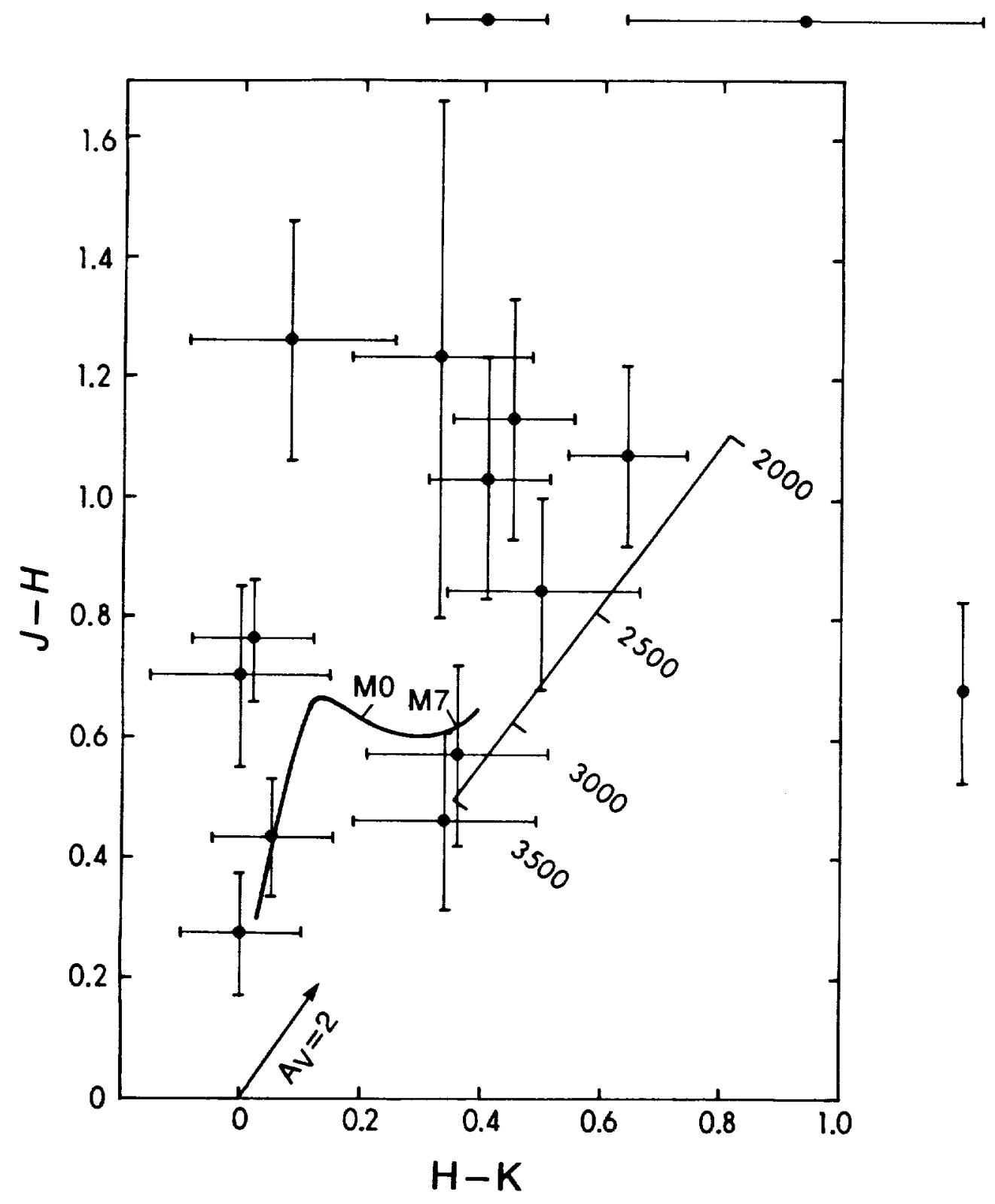

Figure 2 Near infrared color-color plot of the objects (data points with error bars) found in our Taurus survey. The data is from table 1. A 10,000 $\mathrm{K}$ star such as Vega $(\alpha \mathrm{Lyr})$ define the zero point of $\mathrm{H}-\mathrm{K}$ and $\mathrm{J}-\mathrm{H}$ colors. Also shown is a vector corresponding to $A_{V}=2$ mag of interstellar reddening in $\mathrm{H}-\mathrm{K}$ and $\mathrm{J}-\mathrm{H}$. The curve with a J-H peak near $\mathrm{H}-\mathrm{K}=0.15$ represents the average colors of main sequence stars from Probst and Liebert (1983 ApJ, 274, 245) with the approximate positions of $M O$ and $M 7$ spectral types indicated. The straight line with tick marks shows the infrared colors of black bodies with temperatures of 2000 to $3500 \mathrm{~K}$ as indicated. The points outside the box represent stars with only H-K (top) or J-H (right side) measurements. Exceptionally red objects, toward the top right in this figure, are suggested to be possible low mass brown dwarfs in the Taurus association. 